\title{
Posterior Fossa Intradiploic Epidermoid Cyst: A Case Report
}

\author{
Ng WP, Liew BS, Gee TS, Azmin KR \\ Department of Neurosurgery, Hospital Sungai Buloh, Malaysia \\ ABSTRACT
}

\begin{abstract}
Epidermoid cysts are rare, benign congenital tumours of ectodermal origin which typically present between the third to fifth decade. These tumours comprise approximately $0.2-1.8 \%$ of all intracranial tumours. Though these pearly tumours are potentially curable, subtotal resection may lead to catastrophic complications such as recurrence, granulomatous meningitis and carcinomatous degeneration of cyst wall. We herein report the case of a 36-year-old man who presented with an unusual mixed density posterior fossa epidermoid cyst on imaging studies. Total removal not only cures both tumour and seizure attack in this case but also preserves patient's neurological function.
\end{abstract}

\section{KEY WORDS: Epidermoid cyst, posterior fossa, intradiploic tumour INTRODUCTION}

Epidermoid cysts arise from misplaced inclusion of epithelial remnants that was trapped during neural tube closure between the $3^{\text {rd }}$ and $5^{\text {th }}$ week of embryogenesis. ${ }^{1}$ The cysts have a central core of keratin in concentric layer, desquamated cells, water and cholesterol, lined with stratified squamous epithelium. ${ }^{2}$ Continual processes of desquamation and degradation of cell membrane lead to cystic enlargement, therefore producing an insidious and protracted clinical manifestation. ${ }^{1}$ Their most common presentation is a long-standing, painless lump on the head. ${ }^{3} \mathrm{~A}$ correct diagnostic radiological assessment followed by total surgical removal is the ideal treatment. We report a rare case of intradiploic epidermoid cyst and review the related literatures.

\section{CASE REPORT}

A 36-year-old male presented to our hospital with a single episode of unprovoked generalized tonicclonic seizure, which lasted for a few minutes. Further history revealed he had on and off occipital headache for the past 6 months. He denied any trauma, fever, gait instability, syncope attack, visual or hearing problem. Neurological examination of the patient was found to be normal.

Cranial Computed tomography (CT) scan disclosed a mixed density mass with widening of the diploic space in the right occipital bone and a breech in its outer table (Figure 1).

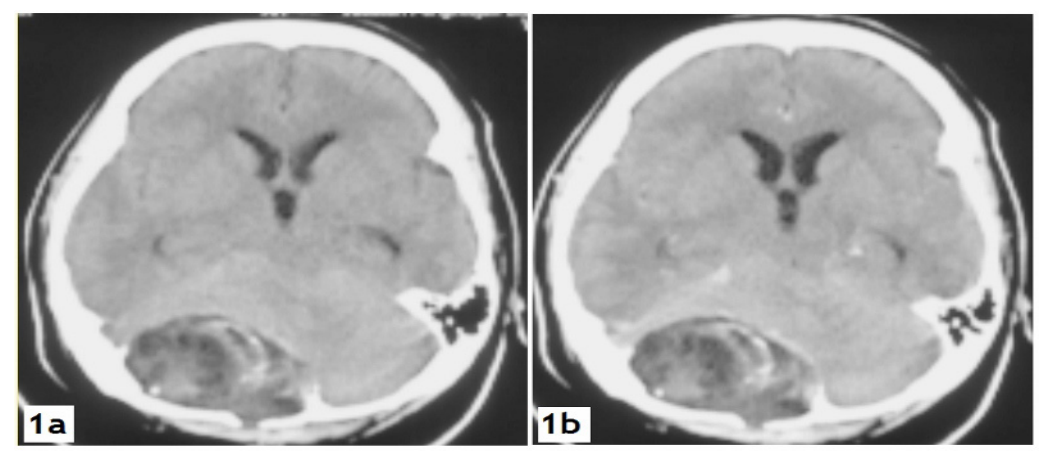

Figure 1. (a) Axial non-contrasted Cranial CT scan shows well circumscribed, lobulated mixed density right posterior fossa mass without surrounding oedema; (b) Axial contrasted Cranial CT scan shows no peripheral or central enhancement of right posterior fossa mass.

Corresponding author:

Dr Ng Wei Ping

Department of Neurosurgery, Hospital Sungai

Buloh, Malaysia

Telephone no: +60124518163

Fax no:+60361454222

E-mail: weipingng@yahoo.com
Cranial magnetic resonance imaging (MRI) scan demonstrated a well encapsulated heterogeneous right posterior fossa intradiploic mass. It demonstrates heterogeneous signal intensity on T1-weighted images, hyperintensity on T2-weigted images and hypointensity on apparent diffusion coefficient (ADC) mapping images. The lesion did not enhance on postgadolinium injection (Figure 2 ). 

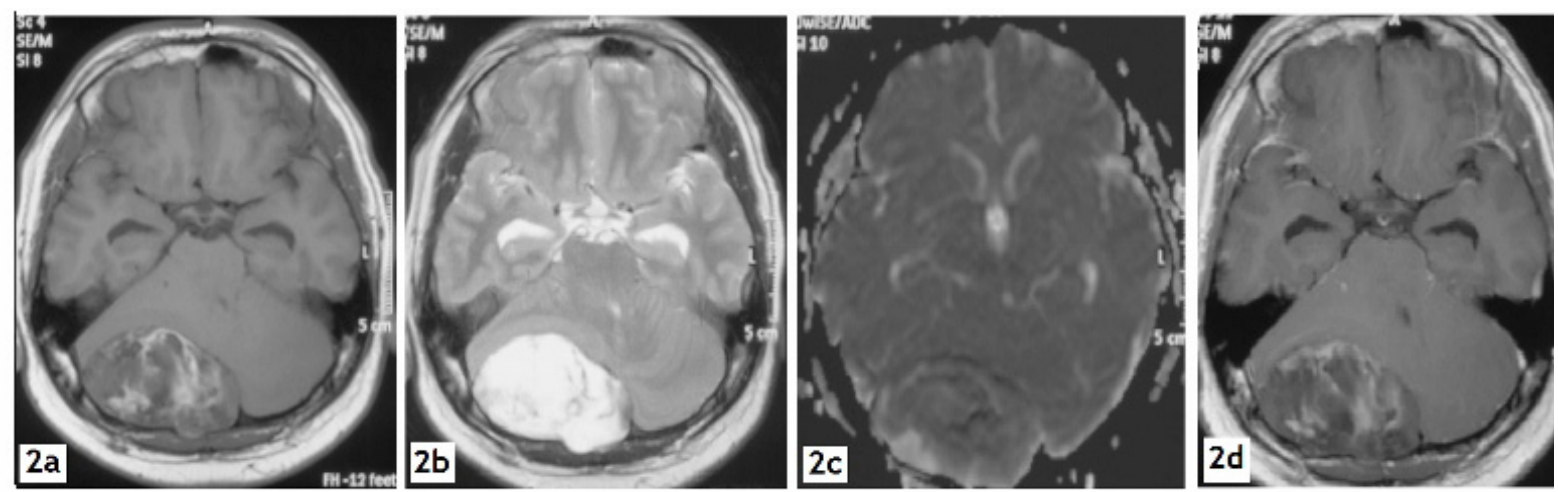

Figure 2. (a) Axial T1-weighted image shows mixed-intensity, off-midline posterior fossa lesion; (b) Axial T2weighted image shows nearly CSF isointense right posterior fossa lesion; (c) Axial ADC map shows the lesion in right posterior fossa appeared hypointense to CSF; (d) Axial T1- weighted post-gadolinium image shows no appreciable enhancement of right posterior fossa lesion.

At surgery, a shiny multilobulated mass herniated through the bony defect when the scalp flap was reflected. The thinned shell like bone flap along with encapsulated tumour was carefully detached and lifted off the underlying dura. Soft, waxy and cheesy white material within a thin cyst wall was identified. There was no hair follicle or other dermal elements seen. Underlying dura was intact and the operative area was thoroughly irrigated at the end of the surgery (Figure 3). Subsequently, reconstruction of skull bone with titanium mesh was performed.
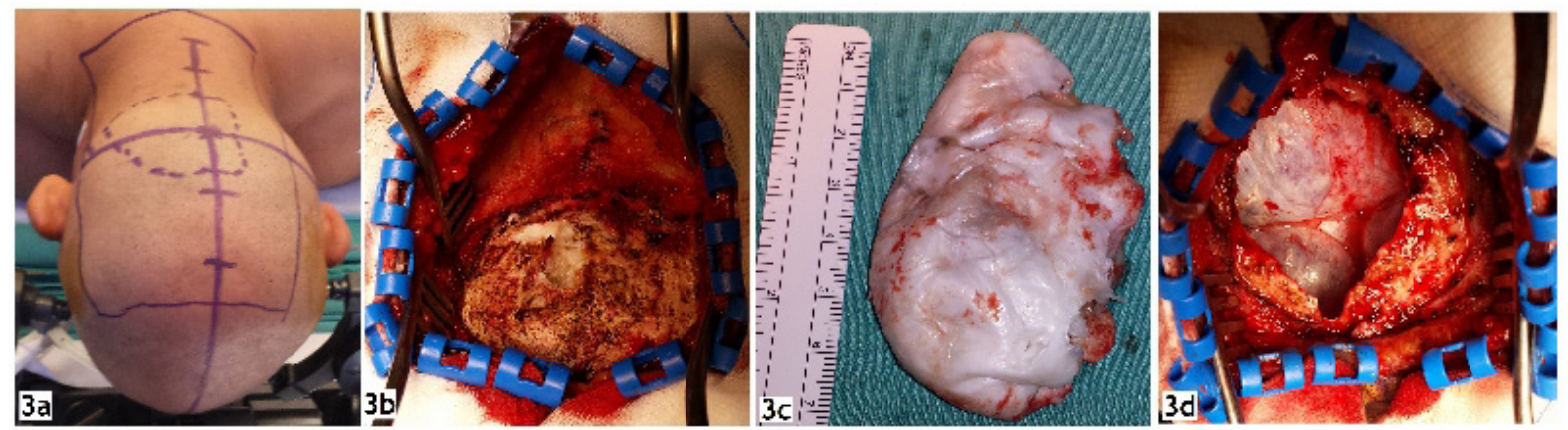

Figure 3. (a) Patient was put in prone position and the anatomical location of the tumour identified; (b) Avascular tumour invading through the thinned shell-like outer table of the occipital bone; (c) Smooth lobulated pearly white tumour contained within a pearly coloured capsule; (d) Craniectomy defect with underlying torcula and intact dura.

Microscopic examination revealed only laminated keratin squames admixed with keratin 'ghost cells' and foci of calcification. The wall of the tumour composed of a keratinizing squamous epithelium and a thin layer of fibrous connective tissue. There was no evidence of previous hemorrhage, brain tissue, sebaceous gland or hair follicle. These findings are consistent with the diagnosis of epidermoid cyst.

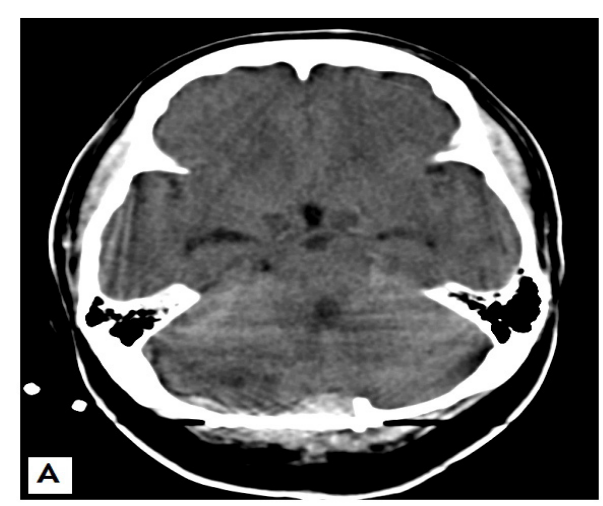

Figure 4. Axial non-contrast cranial CT scan showed complete removal of posterior fossa mass 
The post-operative course was uneventful. Postoperative Cranial CT showed complete removal of the lesion (Figure 4). The patient was discharged without neurological deficit. At 6 months follow up, the patient had complete resolution of seizure and headache.

\section{DISCUSSION}

Epidermoid cysts are known as pearly tumours because of their outer shiny 'mother of pearl' appearance, first described by Cruveilhier. ${ }^{4}$ These cysts are not true neoplasm. They have a slow linear growth rate of one generation per month, similar to normal human skin turnover. ${ }^{5}$ However, they may upon rare occasion undergo malignant degeneration to squamous cell carcinoma. $^{5}$

The cysts present anywhere in the cranial vault, but show a greater tendency to deviate from the midline. $90 \%$ of the tumours are intradural (extra-axial), most frequently involving the cerebellopontine angle and petrous apex followed by parasellar area. ${ }^{6}$ Only a minority of the cysts are located within the intradiploic space. There were 8 cases since the last reported case in $2011 .{ }^{10}$ Subsequently, there were no reported case following a thorough Pubmed search. Inclusion of cell nidi in the secondary vesicles during the $5^{\text {th }}$ week of gestation explains this right paramedian posterior fossa intradiploic lesion. ${ }^{7}$

Epidermoid cysts are typically asymptomatic with the mean presentation age at 40 years old. ${ }^{6}$ The cysts envelop neurovascular structures without displacement. Compression of adjacent neural structures by the cyst however results in focal neurological deficit. ${ }^{2}$ Our patient did not have any cerebellar or brainstem manifestations despite presence of a sizable posterior fossa cyst.

Spillages of cystic content into subarachnoid space can cause communicating hydrocephalus. ${ }^{2}$ Concerned with this, we look at the possibility of aseptic meningitis due to spontaneous cyst rupture in this patient. ${ }^{2}$ However, we believe that the unusual seizure manifestation in our present case was mainly caused by early obstructive hydrocephalus.

The definite diagnosis of epidermoid cyst was suggested by imaging and confirmed by histopathological examination. Differential diagnoses include dermoid cyst, arachnoid cyst, cholesterol granuloma, eosinophilic granuloma and aneurysmal bone cyst. ${ }^{7}$

Cranial CT allows for good assessment of both skull involvement and intracranial extension of the cysts. The characteristic changes of the lytic erosion on the right occipital bone with scalloping clear-cut margin were seen. ${ }^{6}$ Thinning of the bone as well as breakthrough of the cortex of outer and inner tables on CT scan indicates a slow-growing intradiploic cyst in our patient.

The cysts usually appear as well-defined lobulated hypodensity masses due to high level of cholesterol crystal on CT scans. ${ }^{2}$ Although uncommon, hyperdense cyst (referred to as 'white epidermoid') does occur. $^{8}$ It is speculated that hyperdensity appearance is due to increased protein content, saponification of keratinized debris due to calcium soaps, abundance of polymorphonuclear leucocytes, secondary dystrophic calcification, inclusion of blood product and lipid debris. ${ }^{6}$

Proliferative and exudative defensive reaction with recurrent minor leaks of lipidic material through the capsule resulted in high protein content. The linear relationship between CT density and protein concentrations of fluid has been documented. ${ }^{8}$ We believe that high protein concentration and calcification are the leading cause of this unusual CT appearance. This explanation is also supported by patient's histopathological findings.

Due to the avascular nature of its thin wall, epidermoid cyst does not take up contrast although minimal rim enhancement along its periphery has been demonstrated. ${ }^{8}$ Therefore, the presence of internal enhancement is an important feature and indicate inflammation or malignant transformation. ${ }^{5}$

On MRI, epidermoid cysts typically display hypointensity on T1-weighted and hyperintensity on T2-weighted sequences. ${ }^{2}$ However, signal intensities vary depending on the composition of lipids, cholesterol and keratin. In this case, high protein content and mild calcification were the major factors for these rare high signal intensity MR appearances.

Lack of signal suppression on a fluid-attenuated inversion recovery (FLAIR) sequence, high signal intensity on diffusion-weighted imaging (DWI) and hypointensity on ADC maps are the most relevant MRfeatures and help eliminate diagnostic uncertainty. ${ }^{9}$ Hypointense signal on ADC maps seen in this patient is due to motion restriction of proton within the cyst. ${ }^{6}$

Macroscopically, epidermoid cysts are usually located intradurally. ${ }^{2}$ In this patient; a white, slightly fluctuant lesion was identified herniating out of the right occipital bone and extends intracranially at surgery. It is believed that this intradiploic cyst originated from aberrant remnant that becomes trapped after neural tube closure. ${ }^{4}$

Microscopically, epidermoid cysts are lined with keratinizing stratified squamous epithelium and filled with anucleate keratin squammes. ${ }^{1}$ This histological features and absence of dermal elements like hair follicle or sebaceous gland differentiate it from dermoid cyst. ${ }^{2}$

Total removal of the cyst without causing any spillage of the cyst content is the definite treatment. The chemical action of proteinaceous debris on arachnoid membrane will cause aseptic meningitis. ${ }^{2}$ The capsule is the only living and 
growing part of the neoplasm, thus it must be carefully dissected from the bone and dura. Complete removal of the cyst and capsule through a single surgical approach in this patient carries good long term prognosis with permanent cure.

In conclusion, intradiploic epidermoid cysts are benign, potentially curable lesions. However, dense adherence of tumour capsule to adjacent neurovascular structures often cause in incomplete removal. As far, no definite data has yet to prove the effectiveness of adjuvant therapy, thus careful follow up is important to prevent recurrence, abscess formation, bleeding and malignant transformation. ${ }^{8}$

\section{REFERENCES}

1. David F Jimenez, et al. Developmental Anomalies: Arachnoid cysts, dermoid, and Epidermoid. Pediatric Neurosuurgery. In Principles of Neurological Surgery. 2012: 129-135.

2. Hassaeen W, Sawaya R. Epidermoid, Dermoid, and Neuroenteric Cysts. In: Winn HR, ed. Youmans Neurological Surgery: Elsevier Saunders; 2011:1523-8.

3. Khan AN, Khalid S, Enam SA. Intradiploic epidermoid cyst overlying the torcula: a surgical challenge. BMJ case reports. 2011;2011. Epub 2011/01/01.

4. Cruveilhier J. Anatomie Pathologique du Corps Humain. Vol 1. Paris: JB Baillere J B; 1829-1835. p341. Book 2.

5. Hao S, Tang J, Wu Z, et al. Natural malignant transformation of an intracranial epidermoid cyst. Journal of the Formosan Medical Association 2010;109 (5):390-6.

6. Sirin S, Gonul E, Kahraman S, Timurkaynak E. Imaging of posterior fossa epidermoid tumours. Clinical Neurology and Neurosurgery 2005;107 (6):461-467.

7. Ciappetta P, Artico M, Salvati M, Raco A, et al. Intradiploic epidermoid cyst of the skull: Report 10 cases and review of the literature. Acta Neu rochirurgia (Wien) 1990(1-2); 102: 33-37.

8. Daniel Nagasawa, Andrew Yew, Michael Safae, et al. Clinical characteristic and diagnostic imaging of epidermoid tumours. Journal of Clinical Neuroscience 2011;18: 1158-1162.

9. Deniz Bulka, Mehrnaz Didgar, Bart Depreitere, et al. Intradiploic epidermoid cyst: Report of two cases with typical CT and MR imaging features. European Journal of Radiology Extra 2011; 77: 23-27.

10. Yavor Enchev, et al. Post-traumatic giant extradural intradiploic epidermoid cysts of posterior cranial fosa: case report and review of the litherature. Journal Korean Neurosurgery Society 2011;49: 53-57. 